\title{
ALGUNAS INTERROGANTES E INTERPELACIONES A LOS ENCUADRES EN LA CLÍNICA DE NIÑOS CON FUNCIONAMIENTO DE TIPO AUTISTA
}

QUESTIONING THE SETTING: WORKING WITH AUTISTIC FUNCTIONING CHILDREN ALGUNS QUESTIONAMENTOS E INTERPELAÇÕES AOS ENQUADRAMENTOS NA CLINICA DE CRIANÇAS COM TRANSTORNOS DO ESPECTRO AUTISTA

Karina Hackembruch Asociación Uruguaya de Psicoterapia Psicoanalítica Montevideo, Uruguay Correo electrónico: karinahackembruch@gmail.com ORCID: 0000-0001-6166-1812

Recibido: 12/2/2021 Aceptado: 19/3/2021 


\section{Resumen}

La clínica infantil interpela en forma constante nuestra forma de posicionarnos en el espacio psicoterapéutico y las intervenciones que allí podemos instrumentar.

El presente trabajo se centra en pensar y compartir algunas reflexiones e interrogantes sobre la clínica de niños con funcionamiento de tipo autista, buscando articularlas en torno a dos ejes: encuadres y aspectos técnicos.

Al ser una clínica marcada por la sensorialidad y las dificultades en la integración psique-soma, parte de los obstáculos o desafios que plantea a la técnica llevan a pensar y realizar modificaciones en el encuadre. En muchos abordajes con estos pacientes, dichos desafíos me han llevado a dispositivos en los que la interdisciplina y, especialmente, la psicomotricidad han intervenido fuertemente.

Palabras clave: niñez, autismo, encuadre, técnica psicoanalítica.

\section{Abstract}

Clinical work with children constantly challenges the way we position ourselves in the psychotherapeutic space and the interventions we make.

This article shares some thoughts and queries about clinical work with functioning autistic children, regarding two dimensions: framings and technical aspects. As this practice is strongly defined by sensoriality and difficulties in the psyque-soma integration, some of the obstacles and challenges it presents lead us to rethink and modify the setting. Many times, these experiences have led me to adopt an interdisciplinary approach in which psychomotor therapy plays a key role.

Keywords: childhood, autism, framing, psychoanalytic technique.

\section{Resumo}

A clínica de crianças interpela de forma constante nossa forma de nos posicionar no espaço psicoterapêutico e as intervenções que nele podemos realizar.

Este trabalho está focado em pensar e compartilhar algumas reflexões e questões sobre a clínica de crianças com transtornos do espectro autista, procurando articular isso em dois eixos: enquadramentos e aspectos técnicos. Pelo fato desta clínica estar marcada pela sensorialidade e as dificuldades na integração psicossomática, parte dos obstáculos ou desafios que a técnica propõe conduzem a pensar e realizar ajustes no enquadramento. Em muitas abordagens com estes pacientes, esses desafios me conduziram a dispositivos nos que a interdisciplina e, em especial, a psicomotricidade interviram de maneira forte.

Palavras-chave: infância, autismo, enquadramento, técnica psicanalítica. 
Los encuadres y la técnica son componentes de la psicoterapia psicoanalítica en constante interpelación en el quehacer clínico, no tanto en su formulación. Concuerdo con Mariam Alizade (2002) en que «El desmantelamiento del encuadre externo convencional es una realidad contemporánea que se presenta como hecho en sí y que plantea un desafío a la teoría de la técnica psicoanalítica» (pp. 15-16). Este reto se incrementa al pensar en una praxis con niños con funcionamiento de tipo autista, con manifestaciones previas a lo sintomático, del orden del trastorno (Bleichmar, 1988), en el terreno de lo no verbal, corporal.

Me interesaría, entonces, partir de algunos disparadores clínicos para pensar el encuadre en los abordajes terapéuticos de niños con retraimiento autista.

\section{DE ALGUNAS POSIBLES EXTRANJERIAS}

Me referiré aquí a uno de los territorios que puede transitar el psicoterapeuta que trabaja con niños con mecanismos autistas: las sesiones conjuntas psicoterapeuta-psicomotricista en sala de psicomotricidad. Este dispositivo de por sí desaloja al psicoterapeuta de gran parte del encuadre externo: su espacio y sus materiales, y lo lleva a compartir cierta extranjería inicial con el paciente. No contar con el respaldo del encuadre puede conllevar, entre otros, el riesgo de perder «la función estable del encuadre externo como depositaria de ansiedades arcaicas» (Bleger, 1967, apud Alizade, 2002). ¿Se podría también pensar que al trabajar con otro encuadre externo se ponen a circular aspectos superyoicos ligados 
a la formación, a los referentes de cada psicoterapeuta, al marco institucional psicoanalítico? Comparto con Alizade (2002) el anhelo de que el analista se arroje a la piscina de sus intuiciones, se libere de las órdenes recibidas por distintos supervisores o por los textos teóricos y sus certezas, nade en el mar de su encuadre interno, sostenido por el proceso formativo que permitió su gestación, se convierta en un descifrador y en un inventor. Que la tarea analítica se torne lúdica.

Pero, al mismo tiempo, nos preguntamos: en nuestro contexto clínico, ¿cuán extranjero y desertor, cuán cobijado y sostenido se puede sentir un psicoterapeuta al transitar un abordaje conjunto? Sentires e interrogantes que quizás trasciendan esta clínica y este dispositivo formen parte de la identidad psicoterapéutica y sus desafíos, a modo de equilibrio dinámico entre instituido e instituyente. Tomo la voz de Ana Rumi (2005) cuando plantea que

Tal vez toda la historia de nuestra práctica está marcada por el fantasma de una escisión entre una técnica más ortodoxa y una más transgresiva, escisión en la que se corre el riesgo de perder el centro mismo de nuestra práctica. (p. 53)

Este transcurrir psicoterapéutico en otro ámbito y en coterapia con otra disciplina no solo despierta inquietudes e interrogantes, sino que, a su vez, brinda la posibilidad de investir desde lo inaugural. Es decir, a partir de cierta experiencia inicial de desamparo del encuadre externo y a resguardo del interno, el psicoterapeuta irá dotando a los nuevos materiales y espacios de funcionalidades y funciones (en el amplio sentido: psíquicas, de ejercitación pura, sensoriales, simbólicas, etc.).

Este nuevo marco de trabajo convoca, desde la materialidad que ofrece (prismas, espaldar, colchones, túneles, escalera, hamacas, etc.), mayoritariamente al movimiento y a lo sensoriomotor, en contraposición 
al ámbito del consultorio y su mundo objetal, que no invita al despliegue corporal y prioriza en su materialidad la expresión de la simbolización secundaria. Es en esta aparente paradoja sintetizada por ambas disciplinas, entre lo corporal-sensorial y lo verbal-simbólico, donde cobran fuerza este dispositivo y las intervenciones que allí se proponen a través de la mediación. Se promueve o retoma el encuentro con un objeto (sonidos, telas, prismas, túnel, masa, pinturas, etc.) capturado sensorialmente o empleado funcional o figurativamente, buscando que pueda transformarse en mediador para el niño. Es decir, que el objeto pueda actualizar algo del funcionamiento somato-psíquico del paciente, desde el cual la presencia atenta y receptiva de los terapeutas pueda enlazar transferencial, corporal o verbalmente, en busca de simbolizaciones posibles.

Que los objetos presentados por el adulto o encontrados por el niño en el transcurso terapéutico se transformen en mediadores no solo depende de sus cualidades materiales y lo que suscitan, sino de los procesos transferenciales que se puedan poner en juego mediante estos. Cuando se ofrece o acompaña determinada actividad del niño, el psicoterapeuta está atento a la lectura contratransferencial del tipo de angustias puestas en juego (de caída, licuefacción, falta de límites corporales, de fragmentación, etc.). Es importante consignar que la especificidad de la lectura transferencial corresponde al psicoterapeuta, como en cualquier proceso psicoanalítico.

En las sesiones conjuntas en sala, se trabaja con el encuadre temporal de las sesiones de psicomotricidad. Desde este encuadre, se plantea un tiempo inicial, el del ritual de entrada, que propicia el encuentro y la acogida, así como la explicitación de resguardos, a través de la verbalización de las reglas de la sala. A continuación, se desarrolla la sesión con acompañamiento $u$ ofrecimiento de experiencias sensoriomotoras, simbólicas, regladas, etc., y se priorizan en estos pacientes las sensoriomotoras como base para el armado somatopsíquico. Se finaliza con el ritual de despedida, 
que se ofrece como una instancia de descentramiento del movimiento y la emoción, así como también permite transicionalizar la separación.

El rol del psicoterapeuta en las sesiones conjuntas en sala de psicomotricidad se puede operacionalizar desde dos posiciones, según el cuerpo del psicoterapeuta se abstenga o no de entrar en juego. Desde mi experiencia al abordar estos trastornos, priorizo trabajar y acompañar al paciente y al psicomotricista desde el movimiento y con toda la implicancia emocional que esto requiere. Entiendo que esta modalidad posibilita contribuir en mayor medida a la integración corporal y al ensamblaje psíquico. Busca, asimismo, disminuir la dicotomización disciplinaria, ya que tanto el psicoterapeuta como el psicomotricista pueden intervenir mediante la palabra y a través del cuerpo, si bien cuerpo y palabra, acción y representación, son pensados y desplegados desde la especificidad de cada disciplina.

Siguiendo a Silvia Bleichmar (2008), considero que esta posición debería mantenerse en el proceso psicoterapéutico también:

El cuerpo del análisis, del encuadre o el setting, como se dice, no juega su función de mantenimiento si no es habitado por el cuerpo del analista. La atención, antes de estar igualmente en suspenso, es presencia atenta, atención, incluso atenciones de un cuerpo. (pp. 76-77)

El psicoterapeuta intenta bascular entre la acción-hacer y la palabra, entre acompañar y proponer, tomando en cuenta que trabajar con niños con un funcionamiento tan arcaico requiere sopesar cuánto de idéntico y repetitivo, cuánto de novedoso y álter, desplegar en cada encuentro con el paciente. Junto con el psicomotricista, busca rescatar al niño del aferramiento sensorial en el que pueda sumirse por el desmantelamiento psíquico (Meltzer, 1984), engarzando haceres, sensaciones y emociones (de los pacientes, del psicomotricista o de él mismo) con los objetos y las funciones psíquicas que pueda ir convocando la sensorialidad puesta en juego. 
Ambos terapeutas se encuentran disponibles para acoger la expresividad del niño, propiciar la transformación de la sensorialidad en placer sensoriomotriz al integrarlo con aspectos tónico-emocionales, que, a modo de vivencias reiteradas en presencia de un otro que convoca un placer compartido, vayan unificando o armando el yo corporal. El psicomotricista contribuye, desde su saber, a enriquecer las lecturas de lo corporal y favorecer experiencias que contengan la agitación motriz, disminuyan la desorganización y vayan configurando envolturas sensoriales que propicien la integración corporal.

Los coterapeutas van conformando una ritmicidad con sus intervenciones, la secuencia de la sesión, el tono y el ritmo de voz, el estilo de movimiento de cada uno, etc., lo que posibilita la conformación de un espacio continente y confiable. Esta envoltura inicial va a permitir que el niño deposite en forma indiferenciada aspectos de sí en ambos terapeutas. Podemos observar cómo, en principio, el paciente se puede vincular con atributos parciales de ambos terapeutas (pelo, voz, demanda de determinada acción, etc.) de manera indistinta. Puede alternar o seguir a esta modalidad de lazo una percepción más unificada de los técnicos, pero su registro permanece indiferenciado de las particularidades de cada uno de ellos, que funcionan como idénticos, dobles (Haag y Urwand, 1993a, apud Brun, 2009).

En la medida en que el abordaje avance y el niño se vaya vivenciando más integrado, comienza a asignar en forma discriminada diferentes aspectos y funciones a cada terapeuta: búsqueda de intensidad o calma, resguardo de la espalda o acogida de la mirada, voz o movimiento, etc. Todas estas dinámicas corporales que dan cuenta del proceso de psiquización transcurren en la alternancia de interacciones duales o triangulares, al menos desde el punto de vista corporal o de la vivencia del adulto. Coexistirán, así, momentos de encuentro dual, donde uno de los dos terapeutas se mantenga a distancia, sosteniendo este lazo 
libidinal desde el silencio o la quietud; con otros en los que la ritmicidad interactiva incluya a los tres.

El marco de las sesiones conjuntas ofrece, además, el amparo del equipo interdisciplinario del que formen parte los coterapeutas. Dentro de este marco, se irá desplegando la red transferencial que van estableciendo entre ellos hacia el resto del equipo, los padres y el niño.

\section{¿TRASVASES DE ENCUADRES O ACTUALIZACIONES DEL INTERNO?}

A partir de esta y otras experiencias clínicas interdisciplinarias, se va constituyendo un bagaje teórico-técnico que conlleva otro posicionamiento en el consultorio mismo.

Arropar y contener como parte de la técnica no implica, en este caso, solo una referencia a una función simbólica, sino que requiere de objetos tangibles que, convocados por psicoterapeuta o paciente, puedan desplegar estos actos. Es así que telas, mantas, túneles son parte de los materiales del consultorio. ¿Podemos pensar que pueden constituirse en parte del encuadre externo o interno del psicoterapeuta que trabaja con niños con estos funcionamientos? Si bien están disponibles para cualquier paciente dentro del espacio del consultorio, los tengo in mente especialmente en la clínica con autismos, en la que promueven desde la acción específica una función psíquica constitutiva. La incorporación de estos materiales en el consultorio hace a un tránsito interdisciplinario y de sesiones conjuntas en sala de psicomotricidad, así como al trabajo de abordajes grupales en comunidades terapéuticas. Este trasvasar un encuadre externo, propio de otra disciplina o de otro dispositivo, al ámbito del consultorio e internalizarlo como recurso promotor de continentes corporales y psíquicos pone sobre la mesa el tema de cómo materiales 
similares pueden ser usados para funciones similares dentro de técnicas y encuadres diferentes.

Otro mojón clínico que ejemplifica el trasvasamiento de encuadres lo constituye la explicitación en el consultorio de reglas de cuidado mutuo y del material, que toma el modelo de las reglas de la sala de psicomotricidad. Cuando predominan los componentes destructivos o la desorganización masiva, acudo a ellas a manera de resguardo para paciente, psicoterapeuta y espacio de trabajo. Apelo a este fondo de seguridad y organización tomando en cuenta si el niño posee recursos psíquicos acordes para captar su significación o si sus vivencias en tratamiento psicomotriz o en sesiones conjuntas conforman una envoltura de cuidado. Seguramente este recurso se apuntale, además, en la necesidad de introducir una palabra tercerizante, un llamado a un otro internalizado, un par que resguarde desde su palabra y reglas.

\section{ENCUADRE ESTÁ EN LA FRONTERA, Y LAS FRONTERAS SE MUEVEN COMO LAS BANDERAS ${ }^{1}$}

Más allá del dispositivo escogido en cada momento del proceso para trabajar con el paciente en consonancia con sus posibilidades y el funcionamiento que presente, podemos delinear algunos prerrequisitos para el encuadre interno del psicoterapeuta, los cuales conllevan modificaciones a la técnica. Comparto con Alizade (2002) que el encuadre interno «constituye la parte íntima y esencial del marco de todo tratamiento. Consiste en un dispositivo de trabajo incorporado a la mente del analista y a la atmósfera de la sesión» (p. 13).

1 Adaptación de la canción Frontera, de Jorge Drexler (1999). 
Parafraseando a Donald Woods Winnicott (1993), podríamos pensar que parte del encuadre interno requerido para trabajar con estos niños hace que seamos psicoterapeutas lo suficientemente pacientes. Empleo aquí el término paciente en el doble sentido: como referencia a la posibilidad de conectarse con los aspectos más arcaicos de los niños, en una contratransferencia muchas veces en clave sensorial, y, al mismo tiempo, a la de contar con un ritmo que tolere la espera, la monotonía y la inercia. En otras palabras, a partir de la técnica y los recursos de intervención, poder moverse y pensar al niño desde la mediación y la figurabilidad que puede ir adquiriendo la sensorialidad en encuentro con la materia.

Anne Brun (2009) plantea que «El uso de las mediaciones permite iniciar un trabajo terapéutico con esos niños que no han alcanzado aún los procesos de simbolización secundarios vectorizados por las palabras y figurar experiencias sensorio-afectivo-motrices no simbolizadas» (p. 67). Esta autora emplea el concepto de médium maleable de Marion Milner para referirse a aquellos objetos capaces de despertar la figurabilidad a través de su vertiente perceptivo-motriz y mediante su captación sensorial y su manipulación. Milner (1955, apud Brun, 2009) considera que el médium maleable estructura el encuadre, así como irá dando cuenta de los movimientos de la psique del niño y será responsable de iniciar los procesos de simbolización.

También nos pueden asistir en la técnica y permitir pensar el encuadre algunos aportes de Didier Anzieu y Bleichmar. Del primer autor podemos tomar el concepto de envoltura psíquica, ya que, con patologías graves, inicialmente el trabajo es sobre la función continente en sus múltiples capas, que se apuntala sobre la piel y lo corporal, para ir desplegándose luego en el yo y el pensar (Anzieu et al., 1990). En el plano de los continentes y en relación al lenguaje, podemos considerar que, aun empleando palabras, el paciente puede permanecer en un registro no simbólico o de simbolización primaria (Roussillon, 2014); nos movemos, 
entonces, en un registro sonoro, sensorial, envolvente, sin sentido. La intervención sobre los continentes atañe también al empleo, por el paciente o el psicoterapeuta, de determinados materiales concretos que en el vínculo terapéutico pueden adquirir el estatuto de médium maleable (túneles, telas, aros, baúles, etc.).

Los aportes de la autora rioplatense sobre el trabajo con los signos de percepción, a partir de lo propuesto por Sigmund Freud, también resultan muy esclarecedores para guiar la clínica en estos casos. Los signos de percepción se constituyen a partir de fragmentos sensoriales no transcritos, perceptos no ligados, que operan como excesos de excitación no procesables, por lo cual son compelidos a evacuarse compulsivamente. La función del psicoterapeuta, en este caso, será entrelazarlos para propiciar las simbolizaciones ausentes a manera de neogénesis (Bleichmar, 2008). Observamos, así, cómo diferentes aspectos teóricos se nutren de componentes del encuadre y de la técnica y se modifican mutuamente.

En relación al encuadre en el trabajo clínico con pacientes con autismo, Jacqueline Girard-Frésard, Francisco Palacio Espasa y Anne Spillmann hacen hincapié en lo repetitivo e inmutable del espacio, en consonancia con el funcionamiento psíquico de estos pacientes, sobre todo de los que permanecen en un registro más primario (Girard-Frésard et al., 2017). Estos autores señalan que la búsqueda de lo repetitivo se apoya en las dificultades relacionales y de apego que presentan estos niños, por lo cual perceptivamente se van a adherir a determinados elementos del entorno clínico. En el abordaje de estos pacientes se aprecia cómo, frecuentemente, el aferramiento a los bordes, guiado por la sensorialidad táctil y las sonoridades adormecedoras, constituye una primera barrera protectora ante un espacio que se impone en toda su novedad. En niños con un funcionamiento más arcaico, al inicio del proceso, el trabajo de construcción de continentes se desarrolla a partir de lo macroespacial: paredes, aberturas, cantos de muebles ofician sensorialmente con 
esa funcionalidad en su carácter delimitante. El área central, asimilable a los contenidos y lo tridimensional, no puede habitarse, solo puede recorrerse en movimiento: mediante el deambular frenético o en compañía de las estereotipias manuales.

Observamos, entonces, cómo el espacio se ofrece y se toma en tanto que eco del acontecer psíquico, ya que

El niño vive su propio self como desprovisto de envoltura, de interior, como una pura superficie sensible en un mundo uni o bidimensional en el que no se diferencian los espacios psíquicos internos y externos y en el que el selfy los objetos son vividos como cofundidos. (Ferrari, 1997, p. 8)

En la medida en que disminuyan los aspectos persecutorios, la hiperexcitabilidad atenúe su papel como segunda piel (Bick, 1968) y el niño acceda a la tridimensionalidad (Meltzer, 1984), podrá ir tolerando en el devenir psicoterapéutico la construcción de continencias sobre su cuerpo. Este proceso, arduo y extenso, podrá desembocar en la incorporación de los agujeros en su función esfinteriana, de delimitación e intercambio del adentro y el afuera, así como en la posibilidad de identificarse con la función continente (de sensaciones, emociones, representaciones, etc.).

Girard-Frésard et al. (2017) plantean, además, que la repetición y regularidad de las sesiones van a ir instaurando una ritmicidad que va a propiciar un primer investimento de apoyo prepsíquico. El ritmo ya introduce una cesura en el tiempo: las pausas entre sesiones y su frecuencia, lo que incorpora una alternancia que convoca a una primera alteridad, no ligada al otro, sino al espacio y al tiempo. Este primer movimiento no va a estar dirigido al psicoterapeuta, entonces, sino justamente al marco espaciotemporal. Es interesante pensar cómo, muchas veces, en el inicio del tratamiento y sobre todo en aquellos pacientes con un funcionamiento 
más primario, la invariante del espacio es delimitada por ellos. Y esa demarcación en algunos casos no coincide con el límite del consultorio, en tanto que parte de la sesión o toda ella transcurre en la sala de espera, en ambos espacios o en el consultorio pero con la puerta abierta. Así, el niño opera en el espacio al intervenir sus límites de acuerdo con sus vivencias corporales y sus mecanismos o maniobras de defensa, sean del orden más fóbico o persecutorio, más confusional o de aislamiento; $\mathrm{y}$, al mismo tiempo, modifica el encuadre desde el espacio.

Esta invariante del encuadre se transforma en variante para el psicoterapeuta, quien debe adaptarse para acompañar al niño leyendo el sentido que pueda tener para él, así como garantizarle al paciente el control de un nuevo territorio que puede transformarse, entonces, en continente de ansiedades. El encuadre externo en sus aspectos tangibles (básicamente no temporales) deja de ser no-proceso (Bleger, 1967) y pasa a ser parte del proceso en el tratamiento de estos pacientes. Podríamos plantear que, a diferencia de lo que sucede con otros tratamientos, el encuadre en la clínica infantil con autismos nos habla espacialmente desde un comienzo y adquiere cierta mudez con el tiempo. Este decir desde lo espacio-sensorial nos interpela desde el principio y nos invita a moverlo y movernos en el proceso de acompañamiento del paciente.

En contrapartida, el niño necesita dotar al mundo objetal del consultorio, incluido el psicoterapeuta, de cierta inmovilidad o anestesiarlo con su manipulación sensorial atípica y repetitiva, a fin de mantenerse a resguardo emocional, en la lejanía de la no implicancia. Podríamos pensar, entonces, que el psicoterapeuta es al mismo tiempo convocado como parte del no proceso, del encuadre, y del proceso, en la medida en que intenta poco a poco descapturarse de este lugar e ir promoviendo interacciones.

Otro prerrequisito importante tiene que ver con mantener una mirada y una coordinación interdisciplinaria y, si es posible también, trabajar 
desde esta perspectiva, ya que contribuye, y mucho, a la constitución subjetiva de los niños con estos trastornos. Si nos centramos en el encuadre, considero que la frecuencia de las sesiones debería también estar atravesada por lo interdisciplinario. Es decir, que se pueda pensar desde el equipo tratante o desde los diferentes profesionales que trabajen con el niño qué abordajes deben priorizarse en este paciente y, a partir de ahí, definir la frecuencia de cada disciplina, que se irá reviendo a lo largo del proceso. Esta decisión debería tomar en cuenta la edad del niño, su inserción en el ámbito educativo, si la tiene, y las condiciones familiares para sostener los tratamientos en todos sus aspectos y complejidades. Es importante, en este sentido, poder trabajar sobre nuestros rasgos narcisistas, omnipotentes y obsesivos, que seguramente ofrecerán resistencia a ceder lugar a otra disciplina, posponer o hacer concesiones con respecto a la frecuencia de la psicoterapia.

A manera de cierre, pienso que cada uno de estos elementos y situaciones clínicas que van conformando nuestro encuadre interno/externo deberían reposar sobre un tejido lo suficientemente sólido y recurrente como para acompañar el ser psicoterapeuta a lo largo de nuestra vida, a la vez que lo suficientemente maleable como para recibir las suturas, añadidos y remiendos que la clínica nos demande. ${ }^{2}$

$\S$

2 Agradezco a la psicomotricista, psiquiatra, psicoterapeuta y amiga Dra. Graciela Roca por el intercambio y el acompañamiento fluido y fructífero en estos temas desde hace treinta años. Y por su generosidad al leer este trabajo y realizar aportes valiosísimos al respecto. En ella les agradezco también a todas las psicomotricistas con las que trabajé e intercambié y de quienes aprendí. 


\section{REFERENCIAS BIBLIOGRÁFICAS}

Alizade, M. (2002). El rigor y el encuadre interno. Revista Uruguaya de Psicoanálisis, 96, 13-16.

Anzieu, D., Houzel, D., Missenard, A., Enríquez, M., Anzieu, A., Gulllaumin, J., Doron, J., Lecourt, E. y NATHAN, T. (1990). Las envolturas psíquicas. Amorrortu.

Bleger, J. (1967). Psicoanálisis del encuadre analítico. Revista Psicoanálisis, 24(2), 241-258.

Bleichmar, S. (1988). Diagnóstico: Una perspectiva metodológica. En Asociación Escuela Argentina de Psicoterapia para Graduados, Cuestiones acerca de la técnica psicoanalítica con niños y adolescentes (pp. 100-108).

Bleichmar, S. (2009). La fundación de lo inconsciente: Destinos de pulsión, destinos de sujeto. Amorrortu.

Brun, A. (2009). Mediaciones terapéuticas y psicosis infantil. Herder.

Brun, A. y Rousillon, R. (eds.). (2014). Formes primaires de symbolisation. Dunod.

FERRARI, P. (1997). Modelo psicoanalítico de comprensión del autismo $\mathrm{y}$ de las psicosis infantiles precoces. Cuadernos de Psiquiatría $y$ Psicoterapia del Niño y del Adolescente, 23-24, 5-21.

Girard-Frésard, J., Palacio Espasa, F. y Spillmann, A. (2017). Précis de psychothérapie psychanalitique de l'enfant autiste, psychotique et borderline. Èrés.

Rumi, A. (2005). Repensando el encuadre: Proceso y acto analítico. Revista de Psicoterapia Psicoanalítica, 7(1), 49-60.

WinnicotT, D. W. (1993). Los procesos de maduración y el ambiente facilitador. Estudios para una teoría del desarrollo emocional. Paidós. 